\title{
Sonolência excessiva diurna e uso de hipnóticos em idosos
}

José Carlos Souza ${ }^{1}$

Luís Alberto Magna ${ }^{2}$

Thaís Helena de Paula ${ }^{3}$

Recebido: 27/11/2002 Aceito: 7/7/2003

\begin{abstract}
RESUMO
Introdução: A sonolência excessiva diurna (SED) e o uso de hipnóticos aumentam com a idade.

Método: Foram entrevistados 148 idosos, com um questionário estruturado e a escala de sonolência Epworth (ESS), de 11/12/2000 a 2/2/2001. Usaram-se estatísticas descritivas e os testes t de Student, análise da variância, quiquadrado e teste de Pearson.

Resultados: Tinham SED 24\% dos idosos (11 pontos ou mais na ESS). Não houve associação entre a idade e o tempo de residência com SED ( $p=0,10$ e $p=0,37$, respectivamente), o gênero e o grau de instrução $(p=0,30$ e $p=0,27)$.

Não houve associação significativa entre ausência ou presença de SED e uso de hipnótico $(p=0,62)$ e uso de meios alternativos para dormir melhor $(\mathrm{p}=0,65)$. SED foi mais freqüente entre os casados/amasiados $(\mathrm{p}<0,01)$. Houve uma maior proporção de entrevistados que consideraram ter tido muito prejuízo no dia seguinte entre aqueles com $\operatorname{SED}(p=0,05)$. Conclusão: Houve uma alta prevalência de SED, uso de hipnóticos e de meios alternativos para dormir melhor entre os idosos, sem associações significativas entre estas variáveis.
\end{abstract}

Unitermos: Idosos; Distúrbio da sonolência excessiva diurna; Uso terapêutico de hipnóticos; Escala de sonolência Epworth e questionário.

\section{ABSTRACT}

Excessive daytime somnolence and hypnotic use in the elderly

Background: Excessive daytime somnolence (EDS) and hypnotics therapeutic use increase with age.

Methods: A group of 148 elderly residents of a continued-care institution was interviewed by means of a structured questionnaire and also the Epworth sleepiness scale (ESS), from december 11, 2000 to february 02, 2001. There were descriptive statistics and analyzed by means of Student's t-tests, analysis of variance (ANOVA), chi-square and Pearson's Test.

Results: EDS was present in $24 \%$ of the elderly persons of the sample (ESS $>11$ ). There was no association among age and residence time with EDS $(p=0.10$ and $p=0.37$ respectively), gender and instruction grade with EDS $(\mathrm{p}=0.30$ and $\mathrm{p}=0.27$ respectively). EDS wasn't associated with both hypnotic therapeutic use $(\mathrm{p}=0.62)$ and alternative habits to sleep better $(p=0.65)$. The married people showed a higher frequency of $\operatorname{EDS}(p<0.01)$. It was also seen that, decreased levels of daily activity were more frequent in those people with EDS $(p=0.05)$.

Conclusion: There was a high prevalence of EDS, hypnotic and alternative sleep habits usage, without significative associations.

Keywords: Aged; Excessive daytime somnolence; Hypnotic-therapeutic-use; Epworth sleepiness scale - questionnaire.

\footnotetext{
Trabalho vencedor do Prêmio J. J. Barros do IX Simpósio Brasileiro de Sono e II Congresso Paulista de Sono, ano 2002, promovido pelo Departamento de Neurologia da Associação Paulista de Medicina.

1 Psiquiatra; Professor da UCDB - Campo Grande, MS. Mestre em Psicologia e Doutor em Saúde Mental pela Faculdade de Ciências Médicas da Unicamp.

2 Médico e Professor Titular de Genética Médica, Departamento de Genética Médica, Faculdade de Ciências Médicas da Unicamp.
}

3 Psicóloga.

Endereço para correspondência:

Rua Theotônio Rosa Pires, 88, Vila Rosa Pires - Campo Grande, MS - CEP 79004-340

Fone/fax: (0xx67) 325-0990

E-mail: josecarlossouza@uol.com.br

Site: www.ucdb.br/docentes/josecarlos 


\section{Introdução e objetivo}

A sonolência excessiva diurna (SED) é um dos sintomas mais comuns em pessoas idosas (Chokroverty, 1999; Johns, 1994; Chiu et al., 1999). É bem conhecido que a prevalência e a intensidade dos transtornos do sono aumentam com a idade (Chokroverty, 1999; Vgontzas e Kales, 1999; Foley et al., 1999a; Foley et al., 1999b; Yamaguchi et al., 1999; Beullens, 1999; Asplund, 1999). Os fatores causais são: fisiológicos (como as mudanças nos padrões de sono), doenças médicas e psiquiátricas (como a hipertensão e a depressão), farmacológicos (uso indevido e abuso de medicamentos) e sociais (mudanças nas rotinas de atividades, de descanso e dos hábitos de sono-vigília) (Chokroverty, 1999; Vgontzas e Kales, 1999; Foley et al., 1999).

A sonolência é a tendência normal em adormecer, uma conseqüência fisiológica da privação do sono (Culebras, 1996). Quando excessiva (SED), enquadra-se no grupo das dissonias. É de suma importância na saúde pública como um fator de risco para acidentes, problemas interpessoais e diminuição da produtividade e da qualidade de vida (Vgontzas e Kales, 1999; Johnson et al., 1999; Johns e Hocking, 1997; Souza e Guimarães, 1999). O termo sonolência é usado neste estudo no sentido da propensão em cochilar ou adormecer quando a intenção é de se manter acordado; deve ser distinta das sensações subjetivas de cansaço ou fadiga, as quais nem sempre estão relacionadas à sonolência (Chokroverty, 1999; Johns e Hocking, 1997; Johns, 1991; Guilleminault, 1994).

Com o aumento da idade do ser humano, há diminuição do número de horas de sono no período de 24 horas e aumento do número de despertares noturnos e do uso de medicações hipnóticas (Chokroverty, 1999; Asplund, 1999; Guilleminault, 1994; Almeida et al., 1999). Nos idosos institucionalizados, a situação é semelhante (Pollak e Perlick, 1991). As mulheres idosas costumam usar essas drogas mais do que os homens (Guilleminault, 1994).

Os hipnóticos são prescritos mais por médicos nãoespecialistas, em grande escala, semelhante aos antigripais (Souza, 1999; Souza et al., 2001), por vezes como indicação paliativa e não terapêutica. Os tradicionais benzodiazepínicos (BDZ) causam mais reações adversas psicomotoras durante o dia do que os atuais não-BDZ, como o zolpidem, zolpiclone e zaleplon, principalmente se tratando de idosos (Freeman et al., 1996). A prescrição dos hipnóticos deve ser precisa e de curta duração, levando-se em conta os seus efeitos benéficos e colaterais, a etiologia do transtorno de sono presente e, em essencial, a idade do paciente.
Existem algumas alterações específicas do sono com o decorrer da idade, como aumento da atividade autonômica (Guilleminault, 1994) que pode predispor o idoso a um sono de pouca qualidade, como também o ritmo circadiano pulsátil, como evidenciado no aumento dos cochilos diurnos, da fadiga e da tendência ao sono diurno no teste de latência múltipla do sono (MSLT) e em outros testes de sonolência diurna, como o teste de manutenção da vigília (MWT), em que a latência inicial do sono é medida com o sujeito sentado, em um quarto silencioso, tentando se manter acordado (Johns, 1991). Outros testes de sonolência são baseados na pupilometria, os quais são onerosos em demasia. Há testes de desempenho psicomotor e escalas, como a escala de sonolência de Stanford (SSS), que é rápida, simples e avalia os sintomas e as sensações em um período em particular, e as escalas analógicas visuais, que medem o nível geral de sonolência diurna e podem estar relacionadas mais ao sono (Johns, 1991). Objetivou-se estimar a prevalência e a associação da SED com o uso de hipnóticos e meios alternativos para dormir melhor, independentemente de suas etiologias e sem o comprometimento de uma relação de causa e efeito. As variáveis independentes foram: idade, sexo, grau de instrução, estado civil e tempo que reside no asilo. Este estudo faz parte de um censo mais amplo sobre hábitos e distúrbios do sono em idosos institucionalizados.

\section{Material e métodos}

O estudo foi original, realizado no Asilo da Velhice Desamparada Indigente São João Bosco, Campo Grande, MS. Trata-se de uma entidade filantrópica, sem fins lucrativos, sendo o único asilo de idosos da cidade. Foram entrevistados todos os 148 idosos internos, sem nenhum critério de exclusão, com uma média de 75,40 anos de idade (desvio-padrão 10,72; mínimo de 60 e máximo de 101 anos), no período de 11 de dezembro de 2000 a 2 de fevereiro de 2001 . O tempo de permanência no asilo variou de 8 meses a 38 anos (desviopadrão 4,66 e média de 4,58). Este estudo não se ateve a avaliar e discutir os diagnósticos psiquiátricos e outros dos idosos, até porque esses dados raramente são relatados nos parcos prontuários existentes na instituição estudada. Como também, não era o objetivo desta pesquisa avaliar a relação de causa-efeito entre doença mental, sonolência excessiva diurna e uso de hipnóticos.

A entrevistadora foi uma estudante do curso de graduação em psicologia da UCDB, devidamente treinada e supervisionada para tal finalidade, contando com o auxílio do corpo de enfermagem do asilo, para obter melhor 
contato interpessoal e vínculo com os idosos. Os instrumentos foram a ESS e um questionário estruturado baseado e adaptado de Giglio (1988) e Rocha (2000) e voltado aos objetivos do estudo. Considerou-se, para validação do instrumento (questionário estruturado), um estudo piloto realizado em 2001, em uma pesquisa domiciliar ampla com uma amostra representativa da população geral da cidade (Souza et al., 2001), com os mesmos instrumentos, os quais foram devidamente adaptados à população idosa institucionalizada.

A escala de sonolência Epworth (ESS) destina-se a medir a propensão ao sono por um método simples, de baixo custo, rápido e padronizado, permitindo estudos populacionais em larga escala. Ela cobre todas as variedades de propensões ao sono, da maior a menor, com oito questões de situações cotidianas, a saber: sentado e lendo; vendo televisão; sentado em lugares públicos (como sala de aula e igreja); andando de trem, carro ou ônibus por uma hora sem parar; deitando-se à tarde para descansar quando as circunstâncias permitem; sentado e conversando com alguém; sentado calmamente após o almoço sem ter tomado bebida alcoólica; parado na direção de um carro, por alguns minutos, em trânsito intenso (congestionado). A distinção é feita entre adormecer e sentir-se simplesmente cansado. A pessoa deve fornecer uma nota de zero a três, quantificando sua tendência a:

- $\quad$ adormecer em nenhuma chance $=0$;

- $\quad$ pequena $=1$

- $\quad$ moderada $=2$;

- alta chance de cochilar $=3$.

Terá SED quem obtiver o total de 11 ou mais pontos na soma das oito situações descritas. Se o sujeito não esteve recentemente nessas situações, ele é questionado a estimar o quanto elas poderiam ter lhe afetado. A ESS tenta verificar o fato de que as pessoas têm diferentes rotinas diárias, algumas facilitadoras e outras inibidoras do sono diurno. Seus escores significativamente distinguem os grupos de pacientes que têm níveis diferentes de sonolência como medidos pelo MSLT, seu padrão-ouro. Os seus escores foram correlacionados significativamente, com a latência do sono durante o dia medida com o MSLT e à noite, com a polissonografia (PSG) (Johns, 1991; Johns 1992). Enfim, a ESS é um instrumento bastante sensível, simples e confiável para medir a SED em populações gerais e específicas, como a deste estudo com idosos institucionalizados. Possui um elevado grau de consistência interna, medido pelo índice alfa de Cronbach $(0,88)$ (Johns, 1991).

Houve a autorização prévia da direção geral do asilo, do corpo de enfermagem e dos próprios probandos, os quais forneceram consentimento informado, antes das entrevistas. Das variáveis demográficas contidas no questionário aplicado, a escolaridade foi considerada, para fins de preenchimento de número suficiente para análise estatística, em duas categorias, a saber: sem instrução e com instrução até o $2^{\circ}$ grau. Pela mesma razão, o estado civil do entrevistado foi reagrupado em quatro categorias: solteiro, casado/ amasiado, viúvo e separado (esta última englobando também os desquitados e divorciados).

A análise de correlação foi aplicada no cruzamento das variáveis idade, sexo, estado civil, grau de instrução, tempo de permanência no asilo e número de anos que vem tomando remédio para dormir. As quatro últimas coletadas somente entre os entrevistados que indicaram a resposta há mais de 1 ano nas perguntas correspondentes. As comparações de proporções no cruzamento de variáveis de atributo em tabelas de contingência foram feitas pelo teste do $\chi^{2}$ (qui-quadrado). Quando necessário o cruzamento entre variáveis contínuas, utilizou-se o teste de correlação simples de Pearson.

O número de pacientes analisados em cada caso dependeu da anotação de respostas de todos eles ou da pertinência ou não da resposta (há questões cuja resposta foi condicionada a uma resposta afirmativa em questão anterior). No primeiro caso, a perda de resposta foi considerada aleatória e sem influência na estimativa dos parâmetros considerados na análise. Desse modo, porcentagens inferiores a 100\% são decorrentes de pelo menos uma das situações acima, quando calculadas sobre o total da amostra obtida.

\section{Resultados}

Os resultados serão apresentados em tabelas conforme as questões dos instrumentos e dos índices de Epworth para a melhor compreensão; 76\% dos entrevistados declararam não ter usado hipnóticos no último mês, enquanto os restantes se distribuíram, principalmente, entre uso com freqüência inferior a uma vez por semana ou três ou mais vezes por semana. Entre os que utilizaram remédio para dormir, no último mês, $97,5 \%$ o fizeram mediante indicação médica. A idade e o tempo de permanência no asilo não foram fatores que influenciaram, significativamente, a utilização ou não de hipnóticos nem a freqüência com que tal prática foi utilizada ( $p=0,93$ e $p=0,20$ respectivamente) (Tabela 1). Tanto o uso de remédio para dormir quanto a freqüência com que o mesmo foi utilizado apareceram, em maior proporção, entre as mulheres $(p=0,002)$. A escolaridade e o estado civil, por outro lado, não tiveram influência significativa sobre essa prática $(\mathrm{p}=0,88 \mathrm{e}$ $p=0,67$, respectivamente). Dos entrevistados, $74 \%$ não 
Tabela 1 Médias e DP da idade e do tempo que reside no asilo quanto ao uso de hipnóticos, no último mês

\begin{tabular}{|c|c|c|c|c|c|}
\hline \multirow[b]{2}{*}{ Resposta } & \multirow[b]{2}{*}{ Número } & \multirow[b]{2}{*}{ Média } & \multicolumn{3}{|c|}{ Idade $(\operatorname{anos})(p=0,93)$} \\
\hline & & & Desvio-padrão & Mínimo & Máximo \\
\hline Não usa & 113 & 75,24 & 10,47 & 60 & 101 \\
\hline Menos de $1 \mathrm{vez} /$ semana & 14 & 76,50 & 7,96 & 62 & 89 \\
\hline 1 a 2 vezes/semana & 3 & 77,00 & 18,92 & 60 & 92 \\
\hline \multirow[t]{2}{*}{3 ou mais vezes/semana } & 17 & 74,22 & 12,33 & 60 & 97 \\
\hline & & & \multicolumn{3}{|c|}{ Tempo de asilo (anos) $(\mathrm{p}=0,20)$} \\
\hline Resposta & Número & Média & Desvio-padrão & Mínimo & Máximo \\
\hline Não usa & 114 & 4,10 & 3,65 & 0,08 & 23 \\
\hline Menos de $1 \mathrm{vez} /$ semana & 14 & 4,36 & 2,06 & 1 & 7 \\
\hline 1 a 2 vezes/semana & 4 & 3,50 & 2,52 & 1 & 7 \\
\hline 3 ou mais vezes/semana & 18 & 6,11 & 5,19 & 0,08 & 20 \\
\hline
\end{tabular}

utilizaram hipnótico anteriormente; entre os que usaram, a freqüência de uso apresentou proporções semelhantes. A idade não influenciou, significativamente, na utilização de hipnóticos ao longo da vida do entrevistado $(p=0,50)$. Quanto ao tempo de permanência no asilo, no entanto, aqueles que estavam internados há cerca de dez anos (média de 9,86 anos) tiveram tempo total de uso entre seis meses e um ano; entre os demais, o tempo médio de internação foi semelhante $(p=0,03)$ (Tabela 2). As mulheres usaram mais e mais freqüentemente soníferos do que os homens $(p=0,001)$, muito embora o tempo total de uso não tenha se associado à escolaridade e ao estado civil $(\mathrm{p}=0,26 \mathrm{e}$ $\mathrm{p}=0,79$, respectivamente)

Entre os meios alternativos para dormir melhor: banho, isoladamente ou combinado com outra coisa, apareceu em $60 \%$ dos casos, ficando o chá em $2^{\circ}$ lugar com $20 \%$ dos casos. A média de idade e o tempo de permanência no asilo foram semelhantes entre os que fizeram ou não alguma coisa para dormir melhor $(\mathrm{p}=0,99$ e $\mathrm{p}=0,89$, respectivamente). Fazer alguma coisa para conseguir dormir melhor não teve influência significativa do sexo $(\mathrm{p}=0,09)$ nem da escolaridade e do estado civil ( $p=0,90$ e $p=0,82$, respectivamente).

A seguir serão apresentados os resultados da análise estatística decorrente do cruzamento entre a pontuação de Epworth (soma até 10, sem SED; 11 ou mais, com SED) e o total desta pontuação em relação às variáveis demográficas. Tanto a média de idade quanto de tempo de permanência no asilo não estavam associados com a presença ou ausência de SED ( $p=$ 0,10 e $p=0,37$, respectivamente); existiu, no entanto, uma correlação positiva e significativa entre a pontuação na escala de Epworth e a idade $(r=0,23$, $\mathrm{p}<0,01, \mathrm{n}=143$ ), correlação esta que, apesar de significativa, correspondeu a um peso (coeficiente de determinação) de 5,3\% (Tabela 3). Deve-se salientar

Tabela 2 Médias e DP da idade e tempo que reside no asilo quanto ao uso de hipnótico, durante a vida

\begin{tabular}{|c|c|c|c|c|c|}
\hline \multirow[b]{2}{*}{ Resposta } & \multirow[b]{2}{*}{ Número } & \multirow[b]{2}{*}{ Média } & \multicolumn{3}{|c|}{ Idade $($ anos $)(p=0,50)$} \\
\hline & & & Desvio-padrão & Mínimo & Máximo \\
\hline Nunca usou & 111 & 75,36 & 10,50 & 60 & 101 \\
\hline Até 1 mês & 8 & 72,78 & 11,60 & 60 & 89 \\
\hline 1 a 6 meses & 6 & 76,33 & 7,17 & 64 & 83 \\
\hline 6 meses a 1 ano & 7 & 81,86 & 11,74 & 61 & 93 \\
\hline \multirow[t]{2}{*}{ Mais de 1 ano } & 16 & 74,06 & 12,37 & 60 & 97 \\
\hline & & & \multicolumn{3}{|c|}{ Tempo de asilo (anos) $(\mathrm{p}=0,03)$} \\
\hline Resposta & Número & Média & Desvio-padrão & Mínimo & Máximo \\
\hline Nunca usou & 112 & 4,11 & 3,69 & 0,08 & 23 \\
\hline Até 1 mês & 9 & 4,79 & 3,06 & 0,08 & 10 \\
\hline 1 a 6 meses & 6 & 5,50 & 2,88 & 2 & 10 \\
\hline 6 meses a 1 ano & 7 & 9,86 & 13,09 & 1 & 38 \\
\hline Mais de 1 ano & 17 & 4,99 & 4,68 & 0,33 & 20 \\
\hline
\end{tabular}


Tabela 3 Médias e DP da idade e do tempo que reside no asilo quanto à SED

\begin{tabular}{|c|c|c|c|c|c|}
\hline \multirow[b]{2}{*}{ Resposta } & \multirow[b]{2}{*}{ Número } & \multirow[b]{2}{*}{ Média } & \multicolumn{3}{|c|}{ Idade $($ anos $)(p=0,10)$} \\
\hline & & & Desvio-padrão & Mínimo & Máximo \\
\hline sem SED & 110 & 74,44 & 10,65 & 60 & 101 \\
\hline \multirow[t]{2}{*}{ com SED } & 35 & 77,83 & 10,44 & 60 & 98 \\
\hline & & & \multicolumn{3}{|c|}{ Tempo de asilo $(\operatorname{anos})(p=0,37)$} \\
\hline Resposta & Número & Média & Desvio-padrão & Mínimo & Máximo \\
\hline sem SED & 111 & 4,78 & 5,17 & 0,08 & 38 \\
\hline com SED & 36 & 3,97 & 2,70 & 0,08 & 10 \\
\hline
\end{tabular}

que, apesar de significativamente diferente de zero, a correlação encontrada é uma "fraca correlação positiva".

A comparação da média da pontuação na escala de Epworth corroborou os resultados acima, indicando que não houve diferença significativa no que diz respeito ao sexo e à escolaridade $(\mathrm{p}=0,44 \mathrm{e} \mathrm{p}=0,93$, respectivamente), mas evidenciando uma média significativamente superior entre os casados/amasiados $(p<0,02)$, enquanto os demais grupos apresentaram médias semelhantes entre si (Tabela 4).

Não houve associação significativa entre ausência ou presença de SED e uso de remédio para dormir $(p=0,62)$ e fazer alguma coisa para dormir melhor $(p=0,65)$. Houve maior proporção de entrevistados entre aqueles com SED $(p=0,05)$, que consideraram ter tido muito prejuízo no dia seguinte. $\mathrm{O}$ uso de hipnóticos não mudou ao longo da vida, mesmo antes da institucionalização.

\section{Discussão}

Whitney et al. (1998) encontraram 20\% de SED entre 4.578 idosos, em um estudo com a ESS. Tais resultados se assemelham a outros entre adultos jovens sadios (Johns, 1994; Johns e Hocking, 1997; Johns,
1991; Whitney et al., 1998). Johns (1991), no estudo de validação da ESS, apresentou resultados com diferenças significativas nos escores da ESS em relação à idade e ao sexo. Vicario et al. (1997), por outro lado, não detectaram diferenças significativas nos escores da ESS em relação a idade e sexo.

Estudos epidemiológicos com populações específicas de idosos, como os institucionalizados, proporcionam o conhecimento da prevalência e das características sociodemográficas de problemas comuns na prática médica diária, como a insônia e a SED que incomodam, levam a prejuízos e comprometimentos diários ao longo da vida e a uso de hipnóticos, por vezes em demasia. O uso de questionários padronizados e de escalas validadas e sensíveis auxilia neste processo e torna viáveis cada vez mais pesquisas.

Uma vez institucionalizado, o idoso recebe cuidados primários de saúde diuturnamente, que podem prevenir problemas que muitas vezes seriam diagnosticados em fase avançada. Em se tratando do ciclo sonovigília, haverá monitorização de suas alterações, com intervenções e diagnósticos precoces, os quais geram tratamentos especializados, melhorando a qualidade de vida de seus internos.

Tabela 4 Médias e DP do índice de Epworth quanto ao sexo, escolaridade e estado civil

\begin{tabular}{|c|c|c|c|c|c|}
\hline $\operatorname{Sexo}(p=0,44)$ & Número & Média & Desvio-padrão & Mínimo & Máximo \\
\hline Feminino & 67 & 7,30 & 5,49 & 0 & 21 \\
\hline Masculino & 84 & 6,58 & 5,70 & 0 & 22 \\
\hline \multicolumn{6}{|l|}{ Escolaridade $(p=0,93)$} \\
\hline Sem instrução & 91 & 6,76 & 5,06 & 0 & 21 \\
\hline Até $2^{\circ}$ grau & 53 & 6,68 & 6,11 & 0 & 22 \\
\hline \multicolumn{6}{|l|}{ Estado civil $(p<0,02)$} \\
\hline Solteiro & 77 & 6,86 & 5,65 & 0 & 22 \\
\hline Casado/amasiado $(\mathrm{p}<0,02)$ & 7 & 13,83 & 7,36 & 0 & 21 \\
\hline Viúvo & 43 & 6,42 & 5,46 & 0 & 21 \\
\hline Separado & 24 & 6,08 & 4,26 & 0 & 14 \\
\hline
\end{tabular}




\section{Conclusão}

Houve alta prevalência de SED, uso de hipnóticos e de meios alternativos para dormir melhor entre os idosos do Asilo São João Bosco.

São essenciais alguns cuidados na prescrição e na administração dos hipnóticos para os idosos, haja vista que, em geral, são pessoas que fazem uso de polifarmácia, havendo maior risco de efeitos colaterais indesejáveis e de interações medicamentosas farmacodinâmicas e farmacocinéticas negativas, até mesmo fatais. Há de se prescrever uma dose inicial de hipnótico mais baixa possível, aumentando-se, paulatinamente, de acordo com a necessidade e conhecendo-se os outros medicamentos que, porventura, o paciente estiver fazendo uso.

É comum no mercado farmacêutico apresentações medicamentosas, não somente de hipnóticos, de difícil uso e manuseio pelo paciente e seus cuidadores, como exemplo, frascos de soluções líquidas com orifício de saída largo, comprimidos de tamanho tal que dificulta a retirada da embalagem e a sua deglutição, assim como a sua, por vezes pequena, quantidade em cada caixa ou frasco. Convém, ainda, considerar-se a situação socioeconômica do idoso, o qual, muitas vezes, sobrevive com uma pequena aposentadoria e tem outras despesas também essenciais.

Há de se bem indicar, prescrever e avaliar a evolução dos efeitos dos hipnóticos, por curto período de uso, no máximo quatro semanas, visando, em última análise, melhor qualidade de vida dos pacientes.

\section{Agradecimentos}

À diretoria, ao corpo de enfermagem e aos internos do Asilo São João Bosco.

\section{Referências bibliográficas}

Almeida, O.P.; Tamai, S.; Garrido, R. - Sleep Complaints Among the Elderly: Results From a Survey in a Psychogeriatric Outpatient Clinic in Brazil. Int Psychogeriatr 11(1): 47-56, 1999.

Asplund, R. - Sleep Disorders in the Elderly. Drugs Aging 14(2): 91103, 1999.

Beullens, J. - Determinanten van Slapeloosheid bij Relatief Gezonde Ouderen. Een Literatuurstudie. Tijdschr Gerontol Geriatr 30(1): 31-8, 1999.

Chokroverty, S. - Sleep Disorders in Elderly Persons. In: Chokroverty, S. Sleep Disorders Medicine: Basic Science Considerations, and Clinical Aspects. Butterworth-Heinemann, Washington, pp. 401-15, 1999.
Chiu, H.F.; Leung, T.; Lam, L.C.; et al. - Sleep Problems in Chinese Elderly in Hong Kong. Sleep 22(6): 717-26, 1999.

Culebras, A. - Clinical Handbook of Sleep Disorders. Primary Hypersomnias. Butterworth-Heinemann, Boston, 1996.

Foley, D.J.; Monjan, A.A.; Izmirlian, G.; Hays, J.C.; Blazer, D.G. Incidence and Remission of Insomnia Among Elderly Adults in a Biracial Cohort. Sleep 22: Suppl 2:S373-8, 1999.

Foley, D.J.; Monjan, A.; Simonsick, E.M.; Wallace, R.B.; Blazer, D.G. - Incidence and Remission of Insomnia Among Elderly Adults: an Epidemiologic Study of 6,800 Persons Over Three Years. Sleep 22: Suppl 2:S366-72, 1999.

Freeman, H.; Puech, A.J.; Roth, T. - Zolpidem: na Update of its Pharmacological Properties and Therapeutic Place in the Management of Insomnia. Elsevier, Paris, 1996.

Giglıo, S.B. - Estudo da Ocorrência das Queixas de Insônia, de Sonolência Excessiva Diurna e das Relativas às Parassonias na População Adulta da Cidade de São Paulo. EPM, São Paulo, 159p. (Tese, Doutorado em Farmacologia), 1988.

Guilleminault, C. - Narcolepsy Syndrome. In: Kryger, M.H.; et al. Principles and Practice of Sleep Medicine. 2.ed. W.B. Saunders Company, Philadelphia, pp. 549-61, 1994.

Johns, M.W. - A New Method for Measuring Daytime Sleepiness: The Epworth Sleepiness Scale. Sleep 14(6): 540-5, 1991.

JoHns, M.W. - Reliability and Factor Analysis of the Epworth Sleepiness Scale. Sleep 15(4): 376-81, 1992.

Johns, M.W. - Sleepiness in Different Situations Measured by the Epworth Sleepiness Scale. Sleep 17(8): 703-710, 1994.

Johns, M. \& Hocking, B. - Excessive Daytime Sleepiness. Daytime Sleepiness and Sleep Habits of Australian Workers. Sleep 20(10): 844-9, 1997.

Johnson, E.O.; Breslau, N.; Roth, T.; Roehrs, T.; Rosenthal, L. Psychometric Evaluation of Daytime Sleepiness and Nocturnal Sleep Onset Scales in a Representative Community Sample. Biol Psychiatry 45(6): 764-70, 1999.

Pollak, C.P. \& Perlick, D. - Sleep Problems and Institutionalization of the Elderly. J Geriatr Psychiatry Neurol 4(4): 204-10, 1991.

Rocha, F. - Um Estudo com Base Populacional de Hábitos de Sono, Prevalência e Fatores Associados a Insônia. Universidade de Brasília, Brasília, 135p. (Tese, doutorado em Ciências da Saúde), 2000.

SouZA, J.C. - Entendendo a Insônia. Editora Paulus, São Paulo, 1999.

SouZA, J.C.R.P. \& Guimarães, L.A.M. - Insônia e Qualidade de Vida. Editora UCDB, Campo Grande, 1999.

SouZA, J.C.; Reimão, R.; MAgna, L.A. - Insônia e Uso de Hipnóticos na População Geral de Campo Grande, MS. Universidade Estadual de Campinas (UNICAMP), Campinas, 155 p. (Tese, Doutorado em Ciências Médicas), 2001.

Vgontzas, A.N. \& Kales, A. - Sleep and its Disorders. Annu Rev Med 50:387-400, 1999.

Vicario, Y.I.; Platon, M.J.R.; Peralejo, D.C.; Parra, A.B.L.; Sierra, E.J. - Epworth Sleepiness Scale in a Sample of the Spanish Population [Letter to the Editor] Sleep 20(8): 676-7, 1997.

Whitney, C.W.; Enright, P.L.; Newman, A.B.; Bonekat, W.; Foley, D.; QuAN, S.F. - Correlates of Daytime Sleepiness in 4578 Elderly Persons: The Cardiovascular Health Study. Sleep 21(1): 27-36, 1998.

Yamaguchi, N.; Matsubara, S.; Momonoi, F.; Morikana, K.; Takeyama, M.; MaEDA, Y. - Comparative Studies on Sleep Disturbance in the Elderly Based on Questionnaire Assessments in 1983 and 1996. Psychiatry Clin Neurosci 53(2): 261-2, 1999. 